\title{
Simulación dinámica de una alternativa de generación de energía a partir de residuos
}

\author{
Giselle Ramírez Flórez ${ }^{1}$, Luisa F. Giraldo Correa², Natalia Tabares Urrea ${ }^{3}$, \\ Juan C. Osorio Gómez ${ }^{*}$
}

' Universidad del Valle, Facultad de Ingeniería, Programa de Ingeniería Industrial

Calle 13 n.o 100-00, Cali, Colombia.

http://orcid.org/0000-0002-9143-7595

2 Universidad del Valle, Facultad de Ingeniería, Programa de Ingeniería Industrial

Calle 13 n. $100-00$, Cali, Colombia.

http://orcid.org/0000-0002-9298-9092

${ }^{3}$ Universidad del Valle, Facultad de Ingeniería, Programa de Ingeniería Industrial

Calle 13 n. 100-00, Cali, Colombia.

http://orcid.org/0000-0001-6492-3697

${ }^{4}$ Universidad del Valle, Facultad de Ingeniería, Escuela de Ingeniería Industrial, Grupo de Estudio Dinámica de Sistemas, Calle 13 n. 100-00, Cali, Colombia. http://orcid.org/0000-0001-5625-5609

*Autor para correspondencia: juan.osorio@correounivalle.edu.co

\section{RESUMEN}

A nivel mundial, la creciente generación de residuos sólidos corre pareja con una demanda de energía eléctrica que aumenta continuamente. Estos dos problemas en conjunto impactan el ambiente incrementando las emisiones de $\mathrm{CO}_{2}$ a la atmósfera. Para manejar los residuos sólidos se han planteado alternativas como la reutilización, el reciclaje o el reúso. Adicionalmente, se ha propuesto el waste to energy como una alternativa para generar energía a partir de los residuos. En este documento se plantea la comparación de los efectos ambientales asociados a la incineración de residuos (waste to energy) con las alternativas de reúso o reciclaje. El modelo planteado mediante la dinámica de sistemas se valida confrontándolo con la información que se tiene en Colombia sobre los residuos de envases plásticos. El modelo de simulación compara los efectos en cinco indicadores ambientales, encontrándose que dichos indicadores se ven favorecidos en algunos escenarios, pero que es necesario continuar investigando en el tema para tener resultados concluyentes y definitivos. Los resultados y las conclusiones buscan aportar elementos para mejorar los procesos de toma de decisión en este aspecto y abren un camino para continuar la investigación en este tema.

Citation: Ramírez Flórez, G., Giraldo Correa, L.F., Tabares Urrea, N. y Osorio Gómez, J. C., (2017). Simulación dinámica de una alternativa de generación de energía a partir de residuos. Mutis 7(1), 67-77, doi:http://dx.doi. org/10.21789/22561498.1191

Received: Marzo 03, 2016. Accepted: Abril 14, 2016. Published on line: Mayo 1, 2017.

Copyright: @2017 Ramírez Flórez, G., Giraldo Correa, L. F., Tabares Urrea, N.y Osorio Gómez, J. C. This is an open-access article, which permits unrestricted use, distributions and reproduction in any medium, provided the original author and source are credited.

Competing Interests: The authors have no conflict of interest.
Palabras clave: waste to energy, dinámica de sistemas, Colombia, reciclaje, envases plásticos.

Dynamic simulation of an alternative waste to energy

\section{ABSTRACT}

Both problems, the growing solid waste generation and electricity demand continually growing impact the environment by increasing emissions of $\mathrm{CO}_{2}$ to the atmosphere. In relation to the solid waste 
management, alternative as reuse, recycling or reuse have been raised. In addition, Waste to energy appears as an alternative to generate energy from waste. In this paper a comparison of the environmental effects associated with waste incineration (waste to energy) versus alternative reuse or recycling is proposed. The model proposed by system dynamics is validated with information related to plastic packaging waste in Colombia. The model compares the effects on five environmental indicators, finding that such indicators are favored in some scenarios, but is necessary more research on the subject to will be conclusive and definitive results. The results and conclusions provide elements to improve decision-making processes in this area and encourage future researches in the subject.

Keywords: waste to energy, system dynamics, Colombia, recycling, plastic containers.

\section{INTRODUCCIÓN Y REVISIÓN DE LA LITERATURA}

Los rellenos sanitarios son una de las fuentes más importantes de emisiones gaseosas. Las emisiones en los rellenos sanitarios contienen principalmente metano $\left(\mathrm{CH}_{4}\right)$ y dióxido de carbono $\left(\mathrm{CO}_{2}\right)$, de 45 a $55 \%$ y 30 a $40 \%$, respectivamente, ambos considerados gases de efecto invernadero que contribuyen al calentamiento global (Jönsson et al., 2003). La cantidad de residuos sólidos urbanos (RSU) que se generan cada año está aumentando rápidamente, por lo cual la gestión de estos es uno de los temas más relevantes de la sociedad moderna impulsada por la necesidad de hacer frente a una producción total de residuos que, desde 1995, ha tenido un aumento considerable (OECD, 2013).

Ante la crítica situación que viven las ciudades por la creciente cantidad de residuos sólidos urbanos que generan, y con ello, la demanda incesante de nuevos sitios para su disposición final, comienzan a evaluarse diferentes tecnologías para el tratamiento de dichos residuos (Greenpace, 2011).

Una de las opciones consideradas usualmente para disminuir los RSU consiste en la incineración o combustión de estos. La utilización de energía a partir de los residuos se puede dividir en varios grupos, dependiendo de si estos residuos son incinerados o si solo se produce un combustible para una utilización posterior. Uno de los argumentos con que la incineración busca obtener apoyo es que genera una energía que se presenta como renovable. Poletto y Da Silva (2009) señalan que la quema controlada de los RSU, cuando se realiza en instalaciones con dispositivos adecuados de control de la contaminación del aire, es una solución definitiva al problema, que tiene bajo impacto ambiental y se vale de criterios técnicos adecuados.

Por otra parte, la generación de electricidad, calor o biocombustibles a partir de fuentes de energía renovables se ha convertido en prioridad en las estrategias de política energética a escala global (Amigun, Musango y Stafford, 2011). Según Nijkamp y Kourtit (2013), las ciudades consumen el $75 \%$ de la energía producida a nivel mundial y generan el $80 \%$ de las emisiones de $\mathrm{CO}_{2}$; de allí que tenga sentido intentar reducir la emisión de gases provenientes de los residuos mediante el uso de estos para generar energía.

Los residuos han sido identificados como una fuente de energías amigables con el ambiente, y su uso es considerado un método efectivo para reducir las emisiones de gases al ambiente (EI Hanandeh y El Zein, 2011). Visto de esta manera, la waste to energy (WtE) representa una excelente oportunidad para enfrentar dos problemas relevantes de las sociedades actuales: la generación de energía a partir de fuentes renovables y amigables con el ambiente, y la correcta disposición y utilización de los residuos sólidos que se generan permanentemente.

La dinámica de sistemas es una metodología que permite evaluar políticas y comportamientos de los sistemas complejos a mediano y largo plazo mediante el estudio de las variables y sus conexiones. Por medio de la simulación dinámica se pueden estudiar los posibles resultados de las decisiones estratégicas que se tomen en un sistema determinado. Esta metodología contempla los bucles de retroalimentación existentes en los sistemas y permite estudiar los cambios posibles a largo plazo. Ha sido ampliamente aplicada desde su creación, en la década de los cincuenta, y existen múltiples trabajos en diversos campos, tales como la ecología, la economía y la ingeniería, y últimamente se vienen presentando notables desarrollos en temas ambientales y de sostenibilidad. Existen diversos softwares que permiten el desarrollo y evaluación de 
estos modelos, entre ellos los más conocidos son el Vensim, Stella y Powersym.

En términos generales, la dinámica de sistemas (DS) tiene como objetivo capturar las características de los sistemas dinámicos por medio de la modelación y la simulación (Schwaninger y Pérez Ríos, 2008). (Nuhoğlu y Nuhoğlu, 2007) la definen como una metodología para analizar los componentes de un sistema que incluye relaciones de causa-efecto, con formulaciones lógicas, demoras en el tiempo y bucles de retroalimentación.

Respecto al uso de la dinámica de sistemas en temas similares al presentado en este artículo, se puede mencionar a Dyson y Chang (2005), quienes usan la dinámica de sistemas para predecir la cantidad de generación de residuos en una zona urbana de rápido crecimiento, con un caso de estudio en la ciudad de San Antonio (Texas). Bazbauers, Berzina y Davidsen (2014) usan la dinámica de sistemas para evaluar políticas de regulación en la disposición final de los residuos de material de empaque, con el propósito de determinar la eficiencia de esas políticas en relación con la disminución de esos residuos en los rellenos sanitarios. Simonetto, Modro y Dalmolin (2013) utilizan la dinámica de sistemas para evaluar el comportamiento de los residuos generados en Brasil, el efecto del reciclaje y su impacto en la política energética, y brindan elementos que soportan los procesos de toma de decisiones en este aspecto. Por otro lado, Wäger y Hilty (2002) utilizan la dinámica de sistemas para evaluar el comportamiento de los residuos plásticos y proponen un modelo de soporte a la toma de decisiones en este aspecto. Feng, Chen y Zhang (2013) desarrollan un modelo de dinámica de sistemas para evaluar el consumo de energía eléctrica en Beijing y la emisión de $\mathrm{CO}_{2}$ asociada; utilizan para su modelo de simulación el software Stella.

Si bien Ahmad (2012) no evalúa el tema de la generación de energía, presenta un modelo de dinámica de sistemas para la gestión de los residuos sólidos municipales en Delhi, donde explora algunas políticas de gestión de los mismos. Cimren, Bassi y Fiksel (2010) también evalúan políticas sobre la gestión de los residuos sólidos, pero este trabajo se hace en Ohio, Estados Unidos. Finalmente, Dasgupta (2014) presenta un modelo de dinámica de sistemas en el que evalúa la posibilidad de generar energía eléctrica a partir de los residuos sólidos una vez han sido llevados al relleno sanitario. Su trabajo es desarrollado en la ciudad de Bhopal, India.

Esta revisión incluye los más recientes e importantes trabajos en los que se involucra la dinámica de sistemas para la gestión de residuos orientada a la generación de energía, trabajos encontrados en las bases de datos académicas que gozan de mayor reconocimiento. Sin pretender ser exhaustiva, presenta los principales documentos existentes. También conviene mencionar que esta temática se ha venido extendiendo como área de interés, y que en la actualidad se vienen desarrollando importantes proyectos relacionados con la gestión de los residuos y la exploración de alternativas que permitan resolver la creciente problemática vinculada a los mismos.

En este artículo se estudia el impacto ambiental asociado a la generación de energía a partir de los residuos de envases de plástico, y se tomarán los datos específicos de Colombia. Para ello se ha desarrollado un modelo de dinámica de sistemas y se ha hecho una simulación utilizando el software Vensim DSS.

\section{REPRESENTACIÓN SISTÉMICA}

Aracil (1995) propone las siguientes fases para el desarrollo de un modelo de dinámica de sistemas:

\section{Conceptualización del sistema}

2. Construcción del modelo

3. Análisis

La figura 1 ilustra la secuencia de pasos descritos previamente. Las flechas indican pasos hacia atrás en el proceso, e indican que la modelación no consiste en recorrer secuencialmente estas etapas por orden correlativo, sino que en algunas ocasiones es necesario repetir una de ellas para reconsiderar algunos supuestos o variables. Es, por tanto, un proceso de modelado iterativo que combina elementos conceptuales y operativos de la DS (Narváez, 2012) 
Figura 1. Etapas del proceso de modelación de Dinámica de Sistemas (Narváez, 2012)

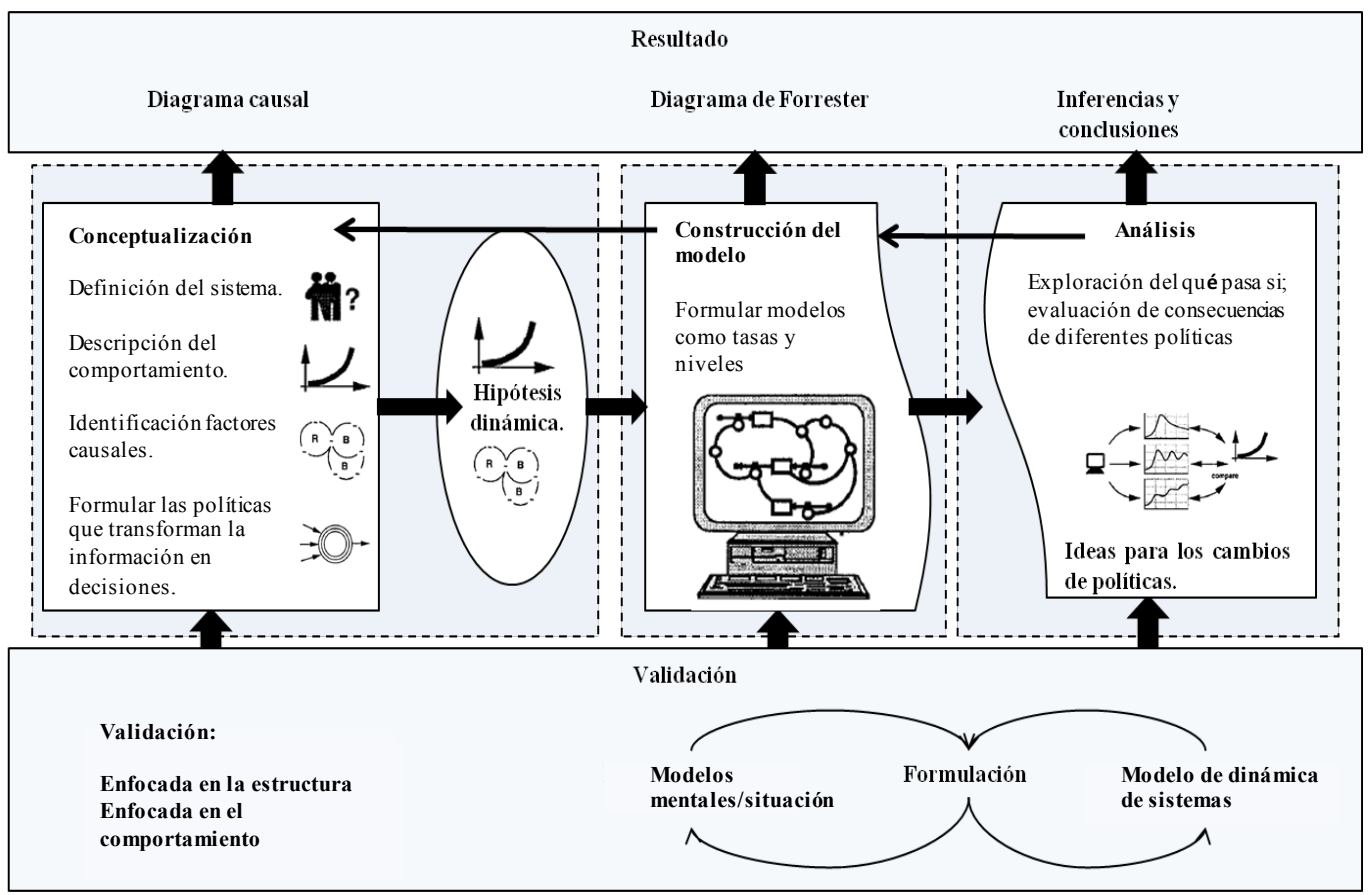

\section{Conceptualización del sistema}

La problemática de estudio es representada mediante el diagrama de influencias que se puede observar en la figura 2, donde se ilustran las principales relaciones causales existentes en el proceso. En este diagrama se aprecian principalmente dos bucles de compensación y uno de refuerzo. El primer bucle de compensación está asociado a los residuos plásticos y a una eventual disminución de estos debido a su aprovechamiento para la generación de energía. El segundo bucle de compensación relaciona las variables de contaminación, regulación, aprovechamiento, residuos y generación de energía. La generación de energía produce mayores niveles de contaminación que promueven el fortalecimiento de la regulación ambiental, pues incentivan y originan ideas de aprovechamiento de los residuos, como, por ejemplo, la generación de energía a partir de estos (waste to energy).

Por otro lado, el bucle de refuerzo representa el potencial de producción de plástico incentivado por el consumo, ligado a la población y su natural crecimiento.

En este diagrama se representan los elementos y relaciones existentes más importantes que permitirán una buena aproximación a la situación real.
Figura 2. Diagrama de Influencias

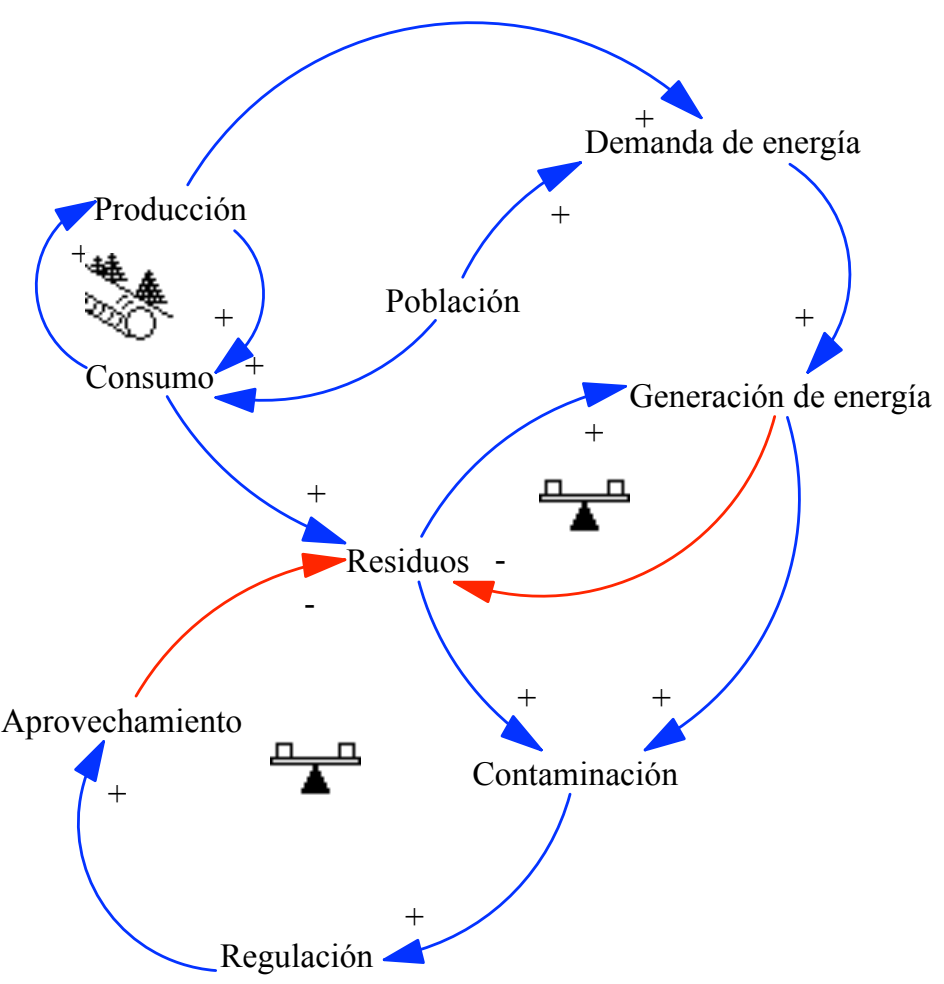




\section{Construcción del modelo}

A partir de la representación causal descrita se construye el diagrama de Forrester, presentado en la figura 3.

Figura 3. Diagrama de Forrester MP: Materia prima. MPV: Materia prima virgen.

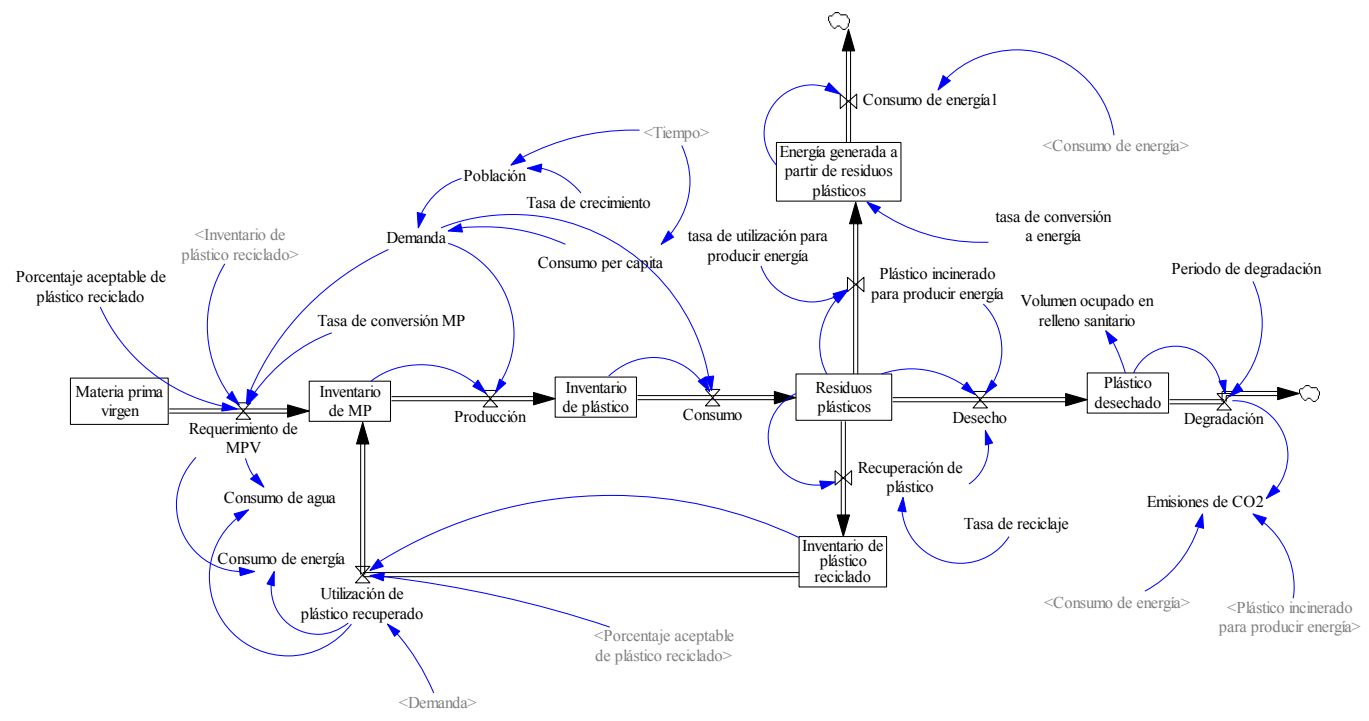

A partir de este diagrama, y utilizando la información relacionada con los residuos de envases plásticos en Colombia, se han realizado la simulación y el modelo. Se contemplan diferentes escenarios mostrados, en la tabla 1 , donde se realizan modificaciones respecto a la tasa de reciclaje, y se expone la proporción de envases de tereftalato de polietileno (PET, por su sigla en inglés) que se utilizarían para la generación de energía.
De esta manera, los escenarios 1 y 2 , junto con el caso "Base del análisis 1", contemplan solamente el reciclaje o reúso sin la opción de incineración. Por su parte, los escenarios 3 y 4 y el caso "Base del análisis 2" contemplan la opción de incineración (waste to energy). Cabe destacar que los análisis del componente 2 considerarán la tasa de reciclaje actual del $24 \%$, según las fuentes oficiales colombianas.

Tabla 1. Escenarios de análisis

\begin{tabular}{cc}
\hline \multicolumn{2}{c}{ Escenarios } \\
\hline Análisis 1: Variación de tasa de reciclaje \\
\hline Base (actual) & $24 \%$ \\
\hline Escenario 1 & $40 \%$ \\
\hline Escenario 2 & $50 \%$ \\
\hline
\end{tabular}

Análisis 2: Variación de la proporción de PET reciclado destinada a generación de energía

\begin{tabular}{cc}
\hline Base & $0 \%$ \\
\hline Escenario 3 & $40 \%$ \\
\hline Escenario 4 & $50 \%$ \\
\hline
\end{tabular}




\section{Análisis}

Respecto a los resultados, se presentan las figuras 4, 5, 6 y 7, relacionadas con los impactos ambientales de la reutilización y reúso, sin considerar la estrategia waste to energy (incineración), solamente variando las tasas de reciclaje.

Figura 4. Comportamiento esperado del consumo de agua ante variaciones en la tasa de reciclaje

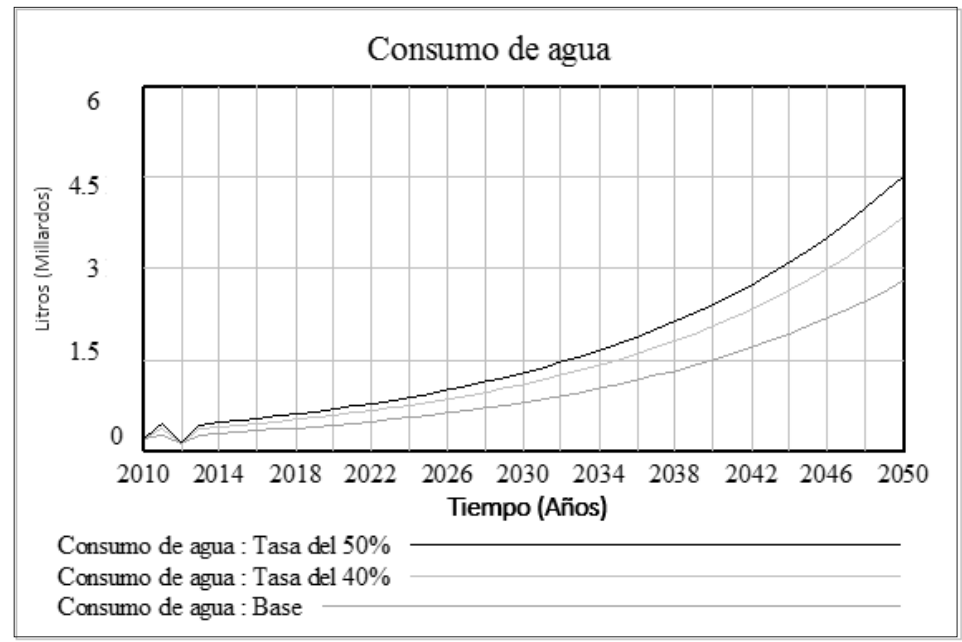

Figura 5. Comportamiento esperado del consumo de energía ante variaciones en la tasa de reciclaje

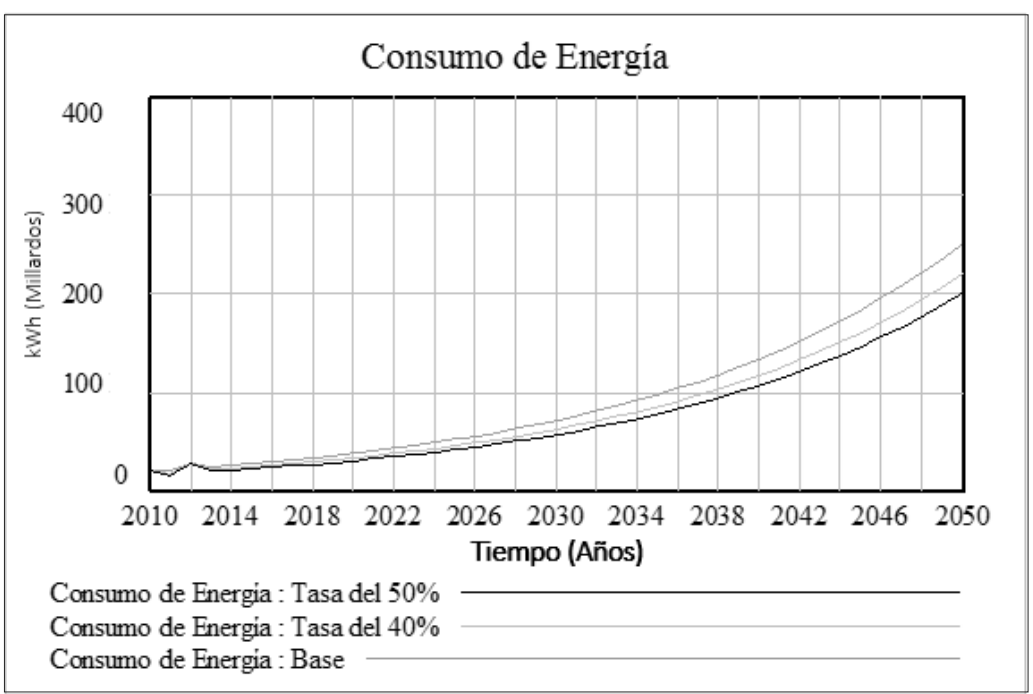


Figura 6. Comportamiento esperado en el tiempo del volumen ocupado en el relleno sanitario ante variaciones en la tasa de reciclaje

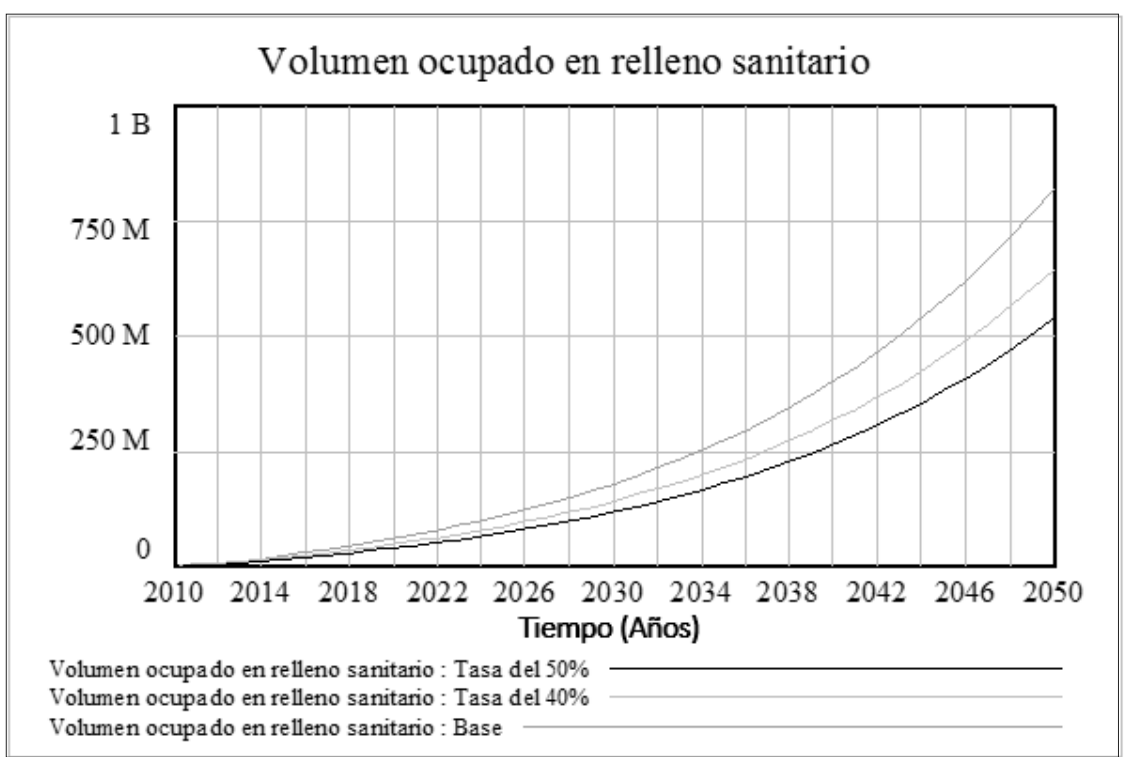

Figura 7. Comportamiento esperado de las emisiones de $\mathrm{CO} 2$ ante variaciones en la tasa de reciclaje

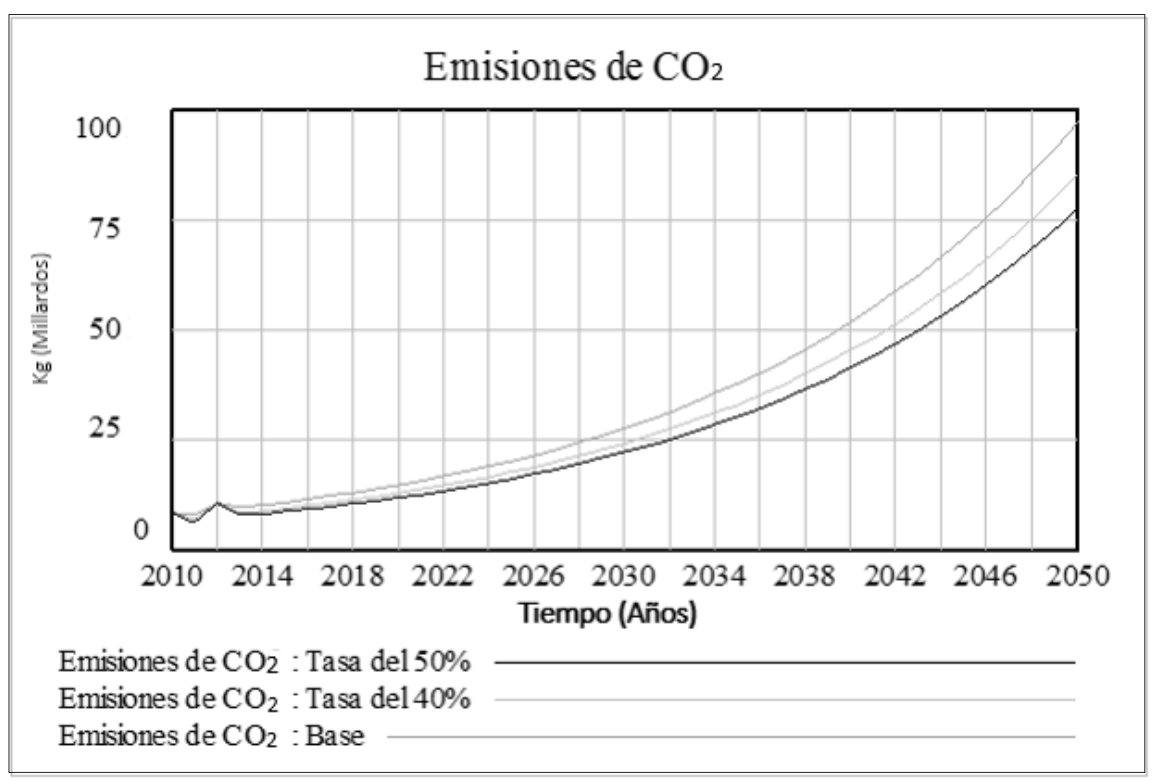

Frente al análisis 1 se aprecia que, a medida que aumenta la tasa de reciclaje, aunque se incrementan los consumos de agua, los otros tres indicadores utilizados muestran disminución. Este aspecto ofrece un punto que debe estudiarse en el futuro, pues si el comportamiento de todos los indicadores que se estén considerando no es el mismo, es decir, si todos au- mentan o disminuyen según el escenario estudiado, surge la necesidad de realizar un análisis global que considere la importancia de cada indicador de acuerdo con el estudio que se está realizando y los indicadores involucrados en el mismo. 
Además, al considerar la importancia y los valores obtenidos por estos indicadores en cada escenario se estará frente a un problema multicriterio que deberá ser abordado con metodologías apropiadas para finalmente poder generar una conclusión global respecto a cuál escenario es el que mejor comportamiento presenta en el sistema.
Sin embargo, como en este artículo se busca explorar la alternativa waste to energy, se presentan los resultados del análisis 2 en las figuras $8,9,10$ y 11 .

Figura 8. Comportamiento esperado del consumo de agua ante variaciones en la tasa de utilización para generar energía

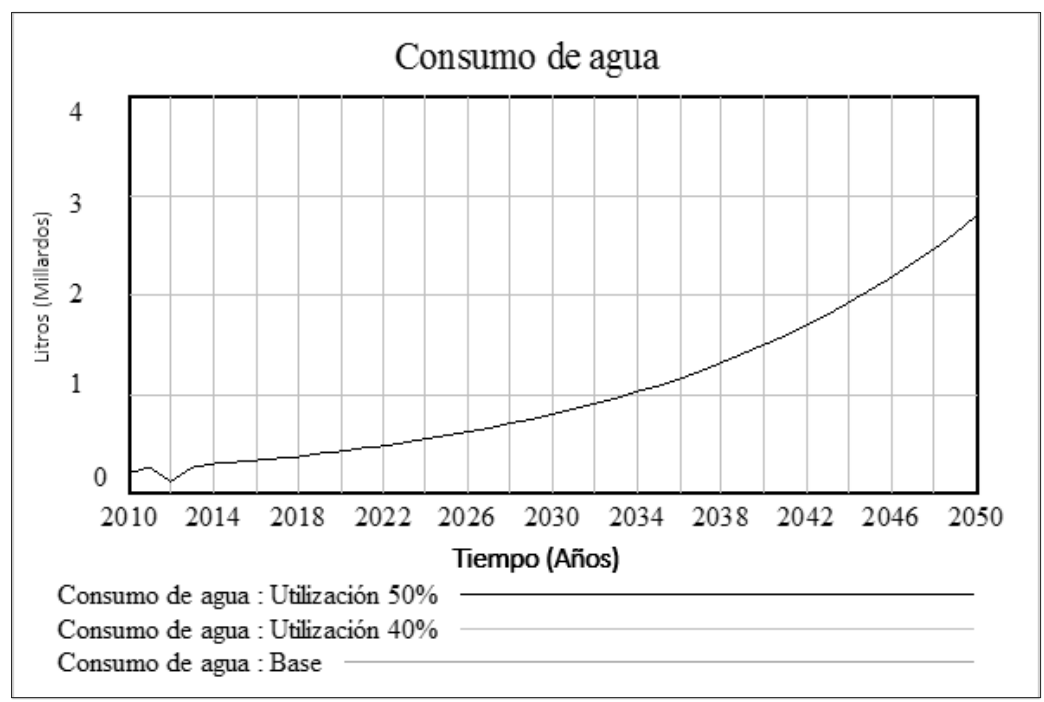

Figura 9. Comportamiento esperado del consumo de energía ante variaciones en la tasa de utilización para generar energía

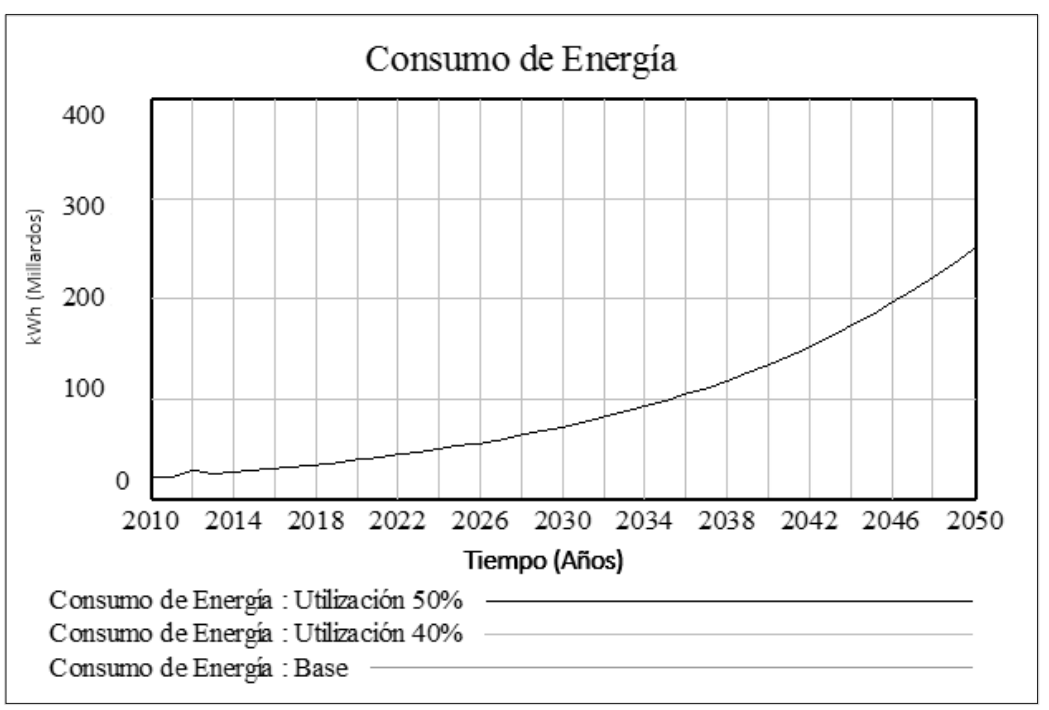


Figura 10. Comportamiento esperado del volumen ocupado en el relleno sanitario ante variaciones en la tasa de utilización para generar energía

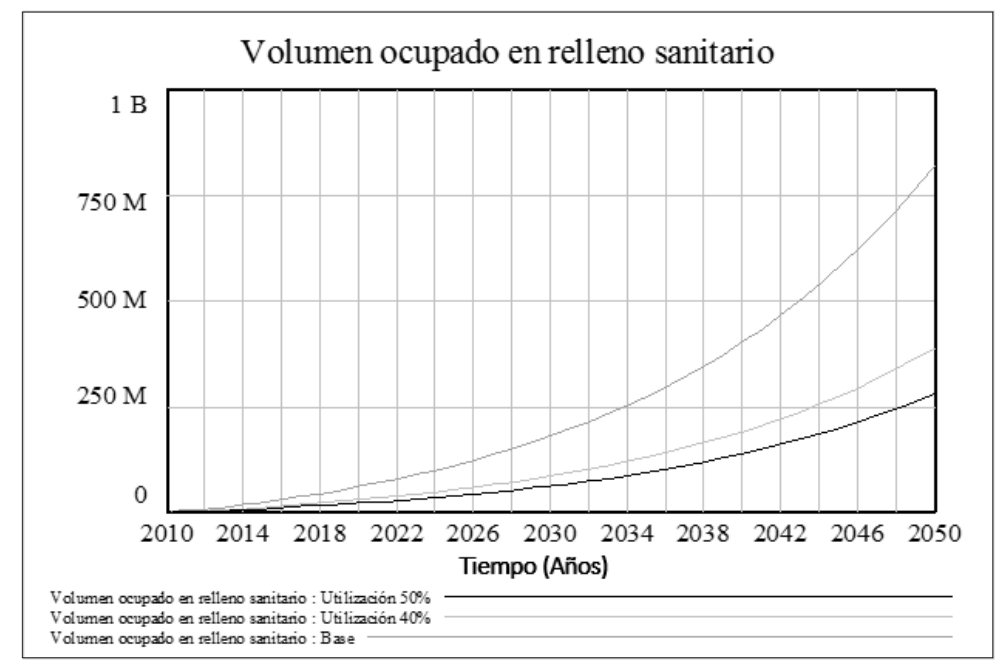

Figura 11. Comportamiento esperado de las emisiones de $\mathrm{CO}_{2}$ ante variaciones en la tasa de utilización para generar energía

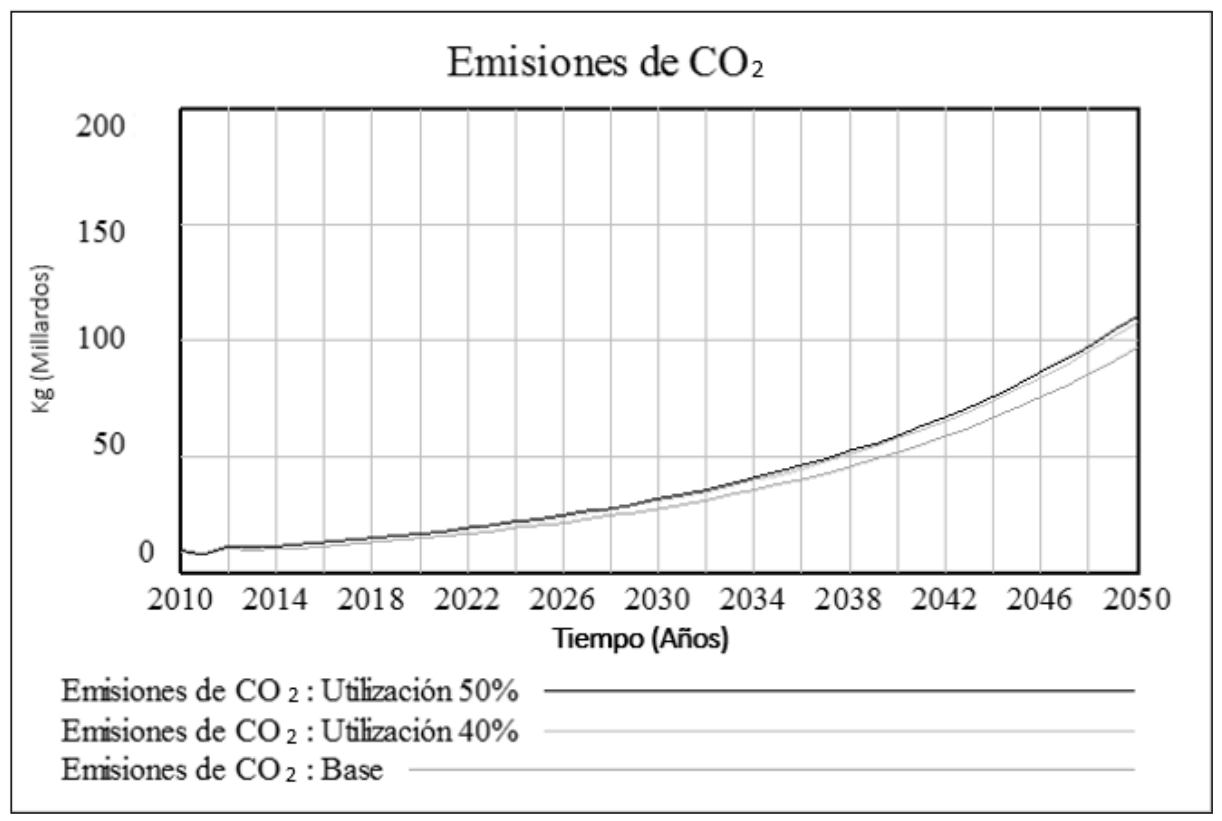

Como se puede apreciar, el consumo de agua y energía no se ve afectado; sin embargo, mientras la tasa de utilización aumenta, el volumen del relleno sanitario disminuye, pero las emisiones de $\mathrm{CO}_{2}$ se incrementan.

Falta, sin embargo, un análisis más, que consiste en evaluar las emisiones de $\mathrm{CO}_{2}$ de la alternativa waste to energy frente a la alternativa de reutilización o reúso al mismo nivel. Se presenta entonces la figura 12, donde se comparan las emisiones para un nivel de reutilización del $40 \%$ en la alternativa de reciclaje, y un nivel de utilización del mismo $40 \%$ para la generación de energía. 
Figura 12. Comportamiento esperado de las emisiones de la alternativa waste to energy frente al reciclaje

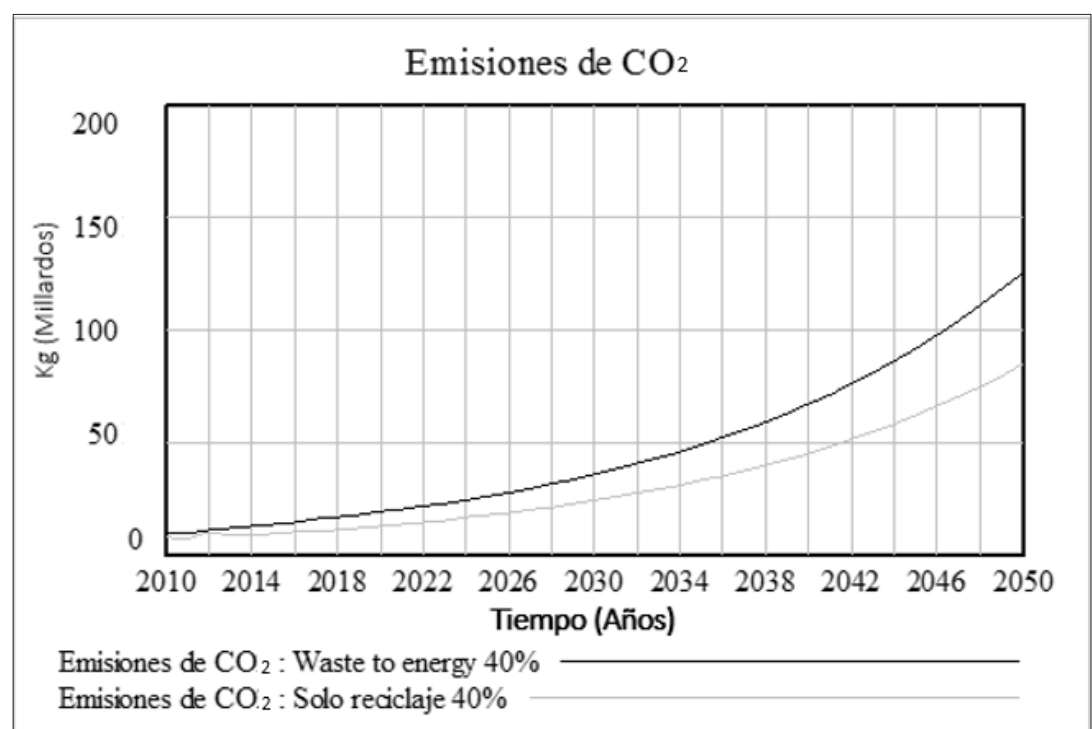

Como se puede apreciar en la figura 12 , las emisiones son mayores para la alternativa waste to energy, por lo cual, aunque no se puede generalizar, se puede afirmar que, respecto al PET, a la luz de las emisiones de $\mathrm{CO}_{2}$ que produce su incineración, es mejor desarrollar estrategias encaminadas a su reutilización, en lugar de utilizarlo para la generación de energía.

Esto quiere decir que, si se considera una alternativa waste to energy aplicada a los envases PET para generar energía eléctrica a partir de su incineración, no será ambientalmente amigable, pues producirá más emisiones de $\mathrm{CO}_{2}$ que si dichos envases se reutilizan. Pero no quiere decir que las estrategias waste to energy no sean apropiadas para otros materiales, como, por ejemplo, los residuos orgánicos, que en Colombia constituyen una parte muy significativa de los residuos sólidos domiciliarios (cerca del $50 \%$ ), lo cual amerita el desarrollo de futuros trabajos relacionados con el tema.

\section{CONCLUSIONES}

La situación abordada en este estudio es un tema de actualidad y sobre el cual existen muchas presiones y discusiones. Con este modelo se pretende hacer una contribución en el proceso de aclarar las mejores decisiones con respecto a los impactos ambientales de alternativas como el waste to energy.

Aunque los resultados presentados no pueden generalizarse, sobre el tema de las emisiones a la atmósfera de $\mathrm{CO}_{2}$ proveniente de la combustión de PET se puede afirmar que es mejor trabajar en la reutilización y el reciclaje de los residuos de este material que usarlos para la generación de energía mediante su incineración.

Respecto a los resultados del análisis 1, se insiste en el estudio multicriterio de los impactos ambientales, pues, como se ha podido apreciar, algunos indicadores muestran una respuesta favorable, mientras que otros demuestran lo contrario.

Finalmente, se propone continuar explorando la alternativa waste to energy desde otras perspectivas, como la eficiencia energética, la contribución en la gestión de los residuos sólidos, y mediante la utilización de otro tipo de residuos, por ejemplo, el componente orgánico de los residuos urbanos. 


\section{REFERENCIAS}

Ahmad, K. (2012). A System Dynamics Modeling of Municipal Solid Waste Management Systems in Delhi. International Journal of Research in Engineering and Technology, 1(4), 628-641.

Amigun, B., Musango, J. K., y Stafford, W. (2011). Biofuels and sustainability in Africa. Renew Sustain Energy, 15(13), 60-72.

Aracil, J. (1995). Dinámica de sistemas (4.a ed.). Publicaciones de Ingeniería de Sistemas. Madrid: Isdefe.

Bazbauers, D. E., Berzina, G., y Davidsen, A. (2014). System dynamics model for analyzing effects of eco-design policy on packaging waste management system. Resources, Conservation and Recycling, 87, 175-190.

Cimren, E., Bassi, A., y Fiksel, J. (2010). T21-Ohio, a system dynamics approach to policy assessment for sustainable development: A waste to profit case study. Sustainability, 2(9), 2814-2832, http:// doi.org/10.3390/su2092814

Dasgupta, T. (2014). Review on energy modeling of urban municipal solid waste management system to the City of Lake, Bhopal. International Journal of Scientific Engineering and Technology, 3(8), 10311035.

Dyson, B., y Chang, N.-B. (2005). Forecasting municipal solid waste generation in a fast-growing urban region with system dynamics modeling. Waste Management (New York, N.Y.), 25(7), 669-79, http://doi.org/10.1016/j.wasman.2004.10.005

El Hanandeh, A., y El Zein, A. (2011). Are the aims of increasing the share of green electricity generation and reducing GHG emissions always compatible ?. Renewable Energy, 36(11), 3031-3036, http://doi. org/10.1016/j.renene.2011.03.034

Feng, Y. Y., Chen, S. Q., y Zhang, L. X. (2013). System dynamics modeling for urban energy consumption and $\mathrm{CO}_{2}$ emissions: A case study of Beijing, China. Ecological Modelling, 252, 44-52, http://doi. org/10.1016/j.ecolmodel.2012.09.008
Greenpace. (2011). Nuevas tecnologías para el tratamiento de residuos urbanos: viejos riesgos y ninguna solución. Publicaciones Greenpace, pp. 38.

Jönsson, O., Polman, E., Jensen, J. K., Eklund, R., Schyl, H., y Ivarsson, S. (2003). Sustainable Gas Enters the European Gas Distribution System. Danish Gas Technology Center.

Nijkamp, P., y Kourtit, K. (2013). The "New Urban Europe": global challenges and local responses in the urban century. Eur. Plan. Stud, 21(3), 291-315.

Nuhoğlu, H., y Nuhoğlu, M. (2007). System Dynamics Approach In Science and Technology Education. Turkish Science Education, 4(2), 91-108.

OECD. (2013). Municipal waste. Factbook 2013: Economic, Environmental and Social Statistics. OECD Publishing.

Poletto, J. A., y da Silva, C. L. (2009). Influencia de la separación de residuos sólidos urbanos para reciclaje en el proceso de incineración con generación de energía. Información Tecnológica, 20(2), 105-112.

Schwaninger, M., y Pérez Ríos, J. (2008). System dynamics and cybernetics: a synergetic pair. System Dynamics Review, 24(2), 145-174, http:// $10.1002 /$ sdr.400

Simonetto, E. D. O., Modro, N. R., y Dalmolin, L. C. (2013). Assessment of Energy Saving in Waste Recycling using System Dynamics. ReA UFSM, 6(2), 319-332, http://doi.org/10.5902/198346597166

Wäger, P. A, y Hilty, L. M. (2002). A Simulation system for waste management - from system dynamics modelling to decision support. Structure, 174-179. 